\title{
Observing Photochemical Transients by Ultrafast X-Ray Absorption Spectroscopy
}

\author{
Melanie Saes, ${ }^{1,2}$ Christian Bressler, ${ }^{1, *}$ Rafael Abela, ${ }^{2}$ Daniel Grolimund, ${ }^{2}$ Steven L. Johnson, ${ }^{3}$ \\ Philip A. Heimann, ${ }^{4}$ and Majed Chergui ${ }^{1, \dagger}$ \\ ${ }^{1}$ Institut de Physique de la Matière Condensée, Université de Lausanne, BSP, CH-1015 Lausanne, Switzerland \\ ${ }^{2}$ Swiss Light Source, Paul-Scherrer Institut, CH-5232 Villigen PSI, Switzerland \\ ${ }^{3}$ Department of Physics, University of California, Berkeley, California 94720 \\ ${ }^{4}$ Advanced Light Source, Lawrence Berkeley National Laboratories, Berkeley, California 94720
}

(Received 18 September 2002; published 31 January 2003)

\begin{abstract}
Accurate determination of the transient electronic structures, which drive photochemical reactions, is crucial in chemistry and biology. We report the detection of transient chemical changes on the picosecond time scale by $x$-ray-absorption near-edge structure of photoexcited aqueous $\left[\mathrm{Ru}(\mathrm{bpy})_{3}\right]^{2+}$. Upon ultrashort laser pulse excitation a charge transfer excited state having a $300 \mathrm{~ns}$ lifetime is formed. We detect the change of oxidation state of the central Ru atom at its $L_{3}$ and $L_{2}$ edges, at a temporal resolution of $100 \mathrm{ps}$ with the zero of time unambiguously determined.
\end{abstract}

DOI: 10.1103/PhysRevLett.90.047403

PACS numbers: 78.70.Dm, 78.47.+p, 82.40.- $\mathrm{g}$

Ultrafast charge transfer processes during photochemical reactions occur in a large class of systems such as small molecules [1], organometallic compounds [2], metalloproteins [3-5], and inorganic media [6], and are in some cases accompanied by ultrafast backtransfers $[3,4]$. X-ray absorption spectroscopy (XAS) is a standard technique for the determination of the static electronic structure [via XANES (x-ray-absorption near-edge structure)] and the local geometric structure [via EXAFS (extended $\mathrm{x}$-ray-absorption fine structure)] around a specific atom. XANES is especially a universal tool to determine the local electronic structure (e.g., oxidation state) of atoms in different chemical compounds [7]. An ultrafast optical pump-X-ray probe experiment using XAS should allow the real-time visualization of both electronic and structural changes. While this approach has been proposed for some time [8-12], to date timeresolved XAS studies have been limited to the ms-ns time domain [13-16], except for one pioneering exception [17].

Several challenges hampered the implementation of XAS with subnanosecond temporal resolution. First, it requires a tunable and monochromatic source of ultrashort x-ray pulses. Second, because of the solvent (background) absorption, solution-phase studies require rather small sample dimensions in order to achieve a reasonable transmission of the $\mathrm{x}$-ray probe beam. Increasing the concentration of the solute, and simultaneously reducing the sample thickness cannot always compensate for this. As a rule of thumb, the number of solute species should not greatly exceed the number of available laser-pump photons per pulse [11], typically $10^{13}-10^{15}$ for commercial amplified femtosecond lasers. Third, the absorption cross sections in the optical and the $\mathrm{x}$-ray domains can differ by up to 3 orders of magnitude and need to be carefully accounted for [11]. Fourth, the low x-ray pulse intensity $\left(<10^{6}\right.$ photons $/ 0.1 \%$ bandwidth per pulse on the sample) requires an extremely sensitive experimental setup. By reducing the measured noise level to nearly the shot noise of the pulsed x-ray source [12] and using a carefully designed sample (accounting for the optical and $\mathrm{x}$-ray absorption cross sections, the available laser pulse energy, and the concentration and thickness of the sample), which minimizes the required number of probing $\mathrm{X}$-ray photons for the highest possible signal-to-noise ratio, time-resolved XAS is possible with existing picosecond and subpicosecond pulsed $\mathrm{x}$-ray sources [11].

In this Letter, we present a time-resolved XANES experiment using $100 \mathrm{ps} \mathrm{x}$-ray pulses from the bend magnet beam line 5.3.1 of the Advanced Light Source (ALS) at the Lawrence Berkeley Laboratories. We are able to take snapshots of the electronic structure of a model complex, ruthenium (II) tris-2, 2'-bipyridine, $\left[\mathrm{Ru}^{\mathrm{II}}(\mathrm{bpy})_{3}\right]^{2+}$, at different time delays after laser excitation. Furthermore, we can scan the time delay at a selected $\mathrm{x}$-ray energy, which also delivers a cross correlation signal between the laser and the $\mathrm{x}$-ray pulses.

$\left[\mathrm{Ru}^{\mathrm{II}}(\mathrm{bpy})_{3}\right]^{2+}$ has served as a paradigm for the ultrafast electron transfer processes in many transition metal complexes [2]. Ru complexes are also excellent reagents for protein modification and internal electron transfer studies [5]. Light absorption by $\left[\mathrm{Ru}^{\mathrm{II}}(\mathrm{bpy})_{3}\right]^{2+}$ results in the formation of a Franck-Condon metal-to-ligand charge transfer singlet excited state, ${ }^{1}$ (MLCT), which undergoes subpicosecond intersystem crossing to a longlived triplet excited state, ${ }^{3}$ (MLCT) [18], with near-unity quantum yield. In the process, the $D_{3}$ symmetry of ground state $\left[\mathrm{Ru}^{\mathrm{II}}(\mathrm{bpy})_{3}\right]^{2+}$ is reduced to the $C_{2}$ symmetry of $\left[\mathrm{Ru}^{\mathrm{III}}\left(\mathrm{bpy}^{-}\right)(\mathrm{bpy})_{2}\right]^{2+}$ in the ${ }^{3}(\mathrm{MLCT})$ state. The reaction cycle is described by the scheme in Fig. 1 (where the superscripts on the left hand side of the ground and excited state compounds denote the spin multiplicity).

The oxidation states of $\mathrm{Ru}^{\mathrm{II}}$ and $\mathrm{Ru}^{\mathrm{III}}$ complexes exhibit pronounced differences in their $L$-edge XANES [7,19,20]. This is shown in Fig. 2(a) for aqueous $\left[\mathrm{Ru}^{\mathrm{II}}(\mathrm{bpy})_{3}\right]^{2+}$ and 


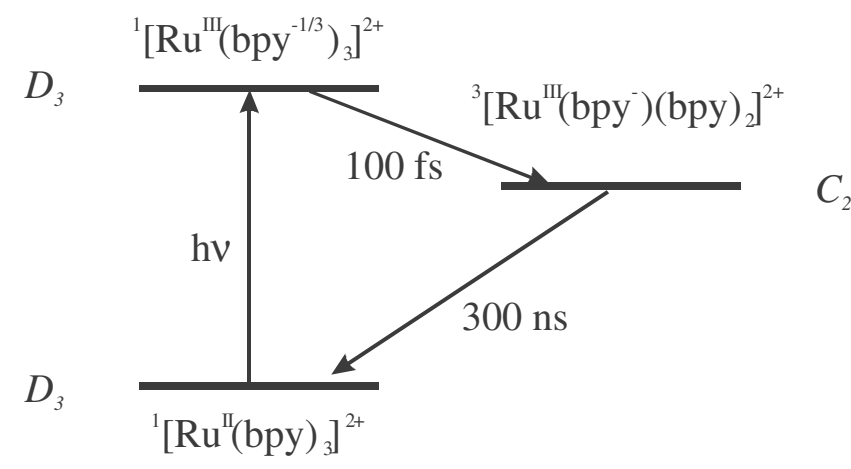

FIG. 1. Photochemical reaction cycle of $\left[\mathrm{Ru}^{\mathrm{II}}(\mathrm{bpy})_{3}\right]^{2+}$.

solid $\left[\mathrm{Ru}^{\mathrm{III}}\left(\mathrm{NH}_{3}\right)_{6}\right] \mathrm{Cl}_{3}$, measured around the $\mathrm{Ru} L_{3}$ edge. A preedge absorption feature at $2841.0 \mathrm{eV}$ [denoted $B$ in Fig. 2(a)] characterizes the bivalent compounds due to the $2 p_{3 / 2} \rightarrow 4 d_{3 / 2}\left(e_{g}\right)$ transition [7], next to the weaker $2 p_{3 / 2} \rightarrow 5 s_{1 / 2}$ transition around $2851 \mathrm{eV}$ (feature $C$ ) [19]. Removal of the weakest bound electron from the fully occupied $4 d_{3 / 2}\left(t_{2 g}\right)$ level generates a trivalent ruthenium compound, opening up an additional absorption due to the allowed $2 p_{3 / 2} \rightarrow 4 d_{5 / 2}\left(t_{2 g}\right)$ (feature $A^{\prime}$ ) in addition to an oxidation state induced shift [7]. Thus we should observe the appearance of the $A^{\prime} / B^{\prime}$ doublet structure in the trivalent $L_{3}$ XANES together with a ca. $1 \mathrm{eV}$ energetic $B \rightarrow B^{\prime}$ and $C \rightarrow C^{\prime}$ shift [Fig. 2(a)], resulting from the change of oxidation state of the Ru central atom and formation of ${ }^{3}$ (MLCT) (Fig. 1).

For this experiment we exploit a special electron bunch filling pattern of the ALS [Fig. 3(a)]. The storage ring is filled with several $2 \mathrm{~ns}$ spaced bunches generating a ca. 550 ns long "superbunch," followed by a ca. $100 \mathrm{~ns}$ dark section, in which one single electron bunch (the "camshaft" pulse) is placed (Fig. 3). This filling pattern allows us to record the intensity of this single $\mathrm{x}$-ray pulse with a suitably fast (and sensitive) avalanche photodiode (APD) detector. The amplified femtosecond laser is synchronized to this camshaft pulse so that the latter follows every laser pulse at $1 \mathrm{kHz}$ repetition rate and at a fixed time delay. For this purpose the 6th harmonic of the fs oscillator repetition rate is synchronized to the radio frequency of the synchrotron $(500 \mathrm{MHz})$ with less than 5 ps time jitter. One of these oscillator pulses (locked to the ALS camshaft pulse) is then amplified at $1 \mathrm{kHz}$ repetition rate, thus maintaining the temporal synchronization. An electronic phase shifter unit allows us to adjust the time delay between the laser and $x$-ray pulses in steps of 5 ps. The single $x$-ray pulses are recorded at twice the laser repetition rate (i.e., at $2 \mathrm{kHz}$ ), and we monitorafter pairwise subtraction of adjacent $\mathrm{x}$-ray pulse intensities - the transient XAS in transmission mode [Fig. 3(c)]. This scheme allows us to improve our detection sensitivity to nearly the shot-noise limit of the $\mathrm{x}$-ray source [12].

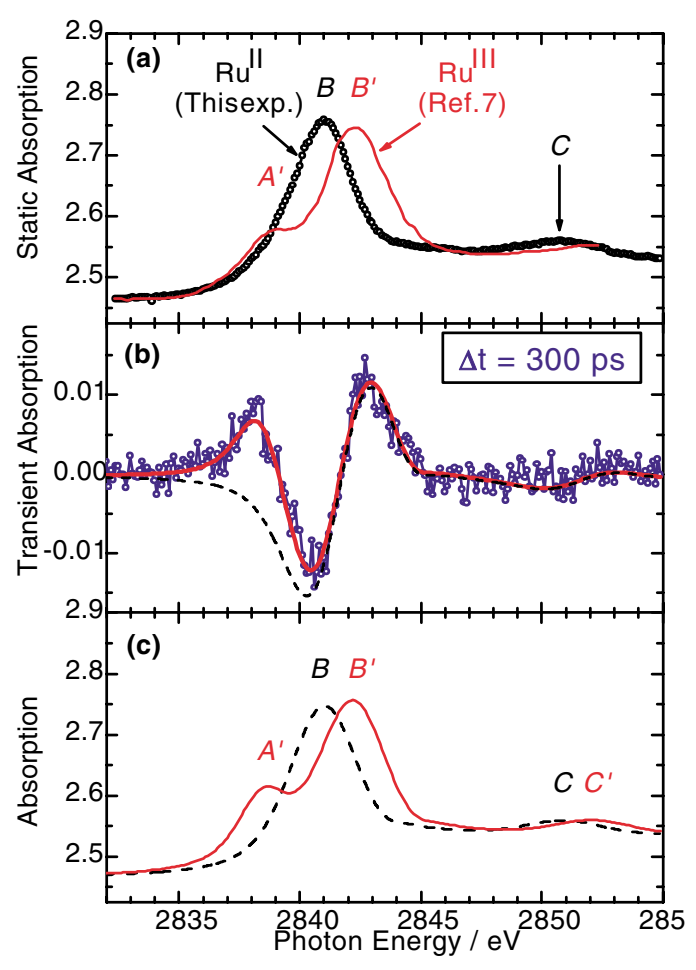

FIG. 2 (color online). (a) Static x-ray absorption spectra at the $\mathrm{Ru} L_{3}$ edge of aqueous $\left[\mathrm{Ru}^{\mathrm{II}}(\mathrm{bpy})_{3}\right]^{2+}$ (open circles, this experiment), and of $\left[\mathrm{Ru}^{\mathrm{III}}\left(\mathrm{NH}_{3}\right)_{6} \mathrm{Cl}_{2}\right]$ (from Ref. [7]). (b) Transient absorption spectrum of photoexcited aqueous $\left[\mathrm{Ru}^{\mathrm{II}}(\mathrm{bpy})_{3}\right]^{2+}$ (open circles) recorded $300 \mathrm{ps}$ after the laser pump pulse. The dashed curve is a fit accounting for the blueshift of the static XAS due to the photoinduced increase of oxidation state, and the solid fit curve includes the appearance of feature $A^{\prime}$ for the reaction intermediate. (c) Reactant $\mathrm{Ru}^{\mathrm{II}}$ state absorption spectrum (dashed) fitted from the experimental data in (a), and intermediate compound absorption spectrum (solid curve) constructed from the reactant state and the transient absorption fit [solid curve in (b)]. The labeled features $A^{\prime}, B^{\prime}, C^{\prime}$, and $B, C$ denote the allowed transitions of $\mathrm{Ru}^{\mathrm{III}}$ and $\mathrm{Ru}^{\mathrm{II}}$, respectively.

We used $80 \mathrm{mmol} / 1$ of $\left[\mathrm{Ru}^{\mathrm{II}}(\mathrm{bpy})_{3}\right]^{2+}$ dissolved in $\mathrm{H}_{2} \mathrm{O}$, which is circulated in a free-flowing jet of $0.1 \mathrm{~mm}$ thickness inside a chamber filled with He. We use $300 \mu \mathrm{J}$, $400 \mathrm{~nm}$ pump pulses from the frequency-doubled output of an amplified Ti:sapphire fs laser system. Both laser and $\mathrm{x}$-ray beams were focused to ca. $0.3 \mathrm{~mm}$ diam on the liquid jet. The X-ray flux was between 500 and 1000 per pulse and $0.1 \%$ bandwidth (depending on the time after injection) at $2.8 \mathrm{keV}$. The flux was derived from a statistical analysis of the pulse height distribution of recorded single $\mathrm{x}$-ray pulses (thus including all subsequent electronic noise contributions), and this represents an effective number of photons per pulse, which contribute to a given pump-probe signal exactly as one would expect from a shot-noise limited source. The so-derived pulse intensity is only slightly less than the expected value at $3 \mathrm{keV}$ (ca. $10^{4}$ per pulse) after correction for various 


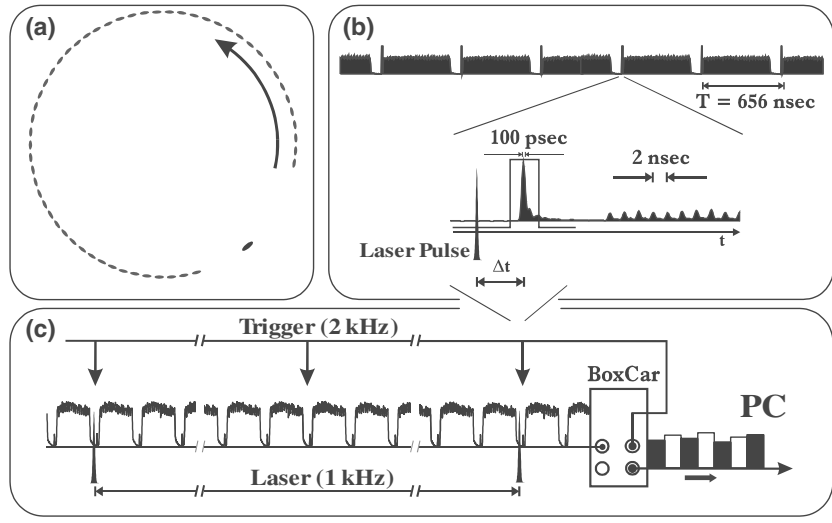

FIG. 3. (a) Schematic of the electron bunch filling pattern at the ALS. Several 2 ns spaced bunches are followed by a ca. $100 \mathrm{~ns}$ long empty section, in which one densely packed electron bunch (called the camshaft pulse) is placed. The emitted radiation (b) is composed of a ca. $550 \mathrm{~ns}$ long "superbunch" together with the well-separated 100 ps long camshaft pulse, to which an amplified fs laser pulse is synchronized with an adjustable time delay $\Delta t$ (see inset). (c) A gated integrator records the camshaft pulse intensity within its ca. $20 \mathrm{~ns}$ long time window [indicated in the inset in (b)] at twice the repetition rate of the fs laser. Thus the output signal of the gated integrator consists of alternating laser-on laser-off $x$-ray intensities, and pairwise subtraction delivers the photoinduced $\mathrm{x}$-ray transmission change.

losses in the beam line (filters, monochromator) and in our experimental setup (He-air gas mixture). The recorded transmission spectra were transformed into absorption spectra via normalization with a transmission spectrum without sample. The spectra were calibrated with standard metal foils to an accuracy of better than $0.3 \mathrm{eV}$.

Figure 2(b) shows the transient absorption spectrum as a function of the x-ray probe energy $E$, recorded 300 ps after laser excitation (open circles). This transient contains all the electronic changes from the reactant state absorption spectrum, $R(E)$, to the product state absorption spectrum, $P(E, t)$, at the time $t$ after photoexcitation. With $f(t)$ being the fraction of excited state species at time $t$, the transient absorption spectrum $T(E, t)$ is given by

$$
T(E, t)=f(t) \cdot[P(E, t)-R(E)] .
$$

Thus we can directly deduce the XANES spectrum of the product state from the measured transient signal and the reactant state XAS, if we know $f(t)$. Alternatively, we can derive $f(t)$, if we know the exact shape of the product state XAS, $P(E, t)$. In this work, we have used different independent methods to derive and crosscheck both $P(E, t)$ and $f(t)$ :

(i) The Ru $L_{2}$ edge (not shown here) consists mainly of the $2 p_{1 / 2} \rightarrow 4 d_{3 / 2}\left(e_{g}\right)$ transition in both bi- and trivalent complexes (features $B$ and $B^{\prime}$ in Fig. 2, respectively), since the $2 p_{1 / 2} \rightarrow 4 d_{5 / 2}\left(t_{2 g}\right)$ transition (feature $A^{\prime}$ ) is for- bidden [7]. Thus the transient XAS of this edge can be unambiguously fitted to Eq. (1) by adjusting the $B \rightarrow B^{\prime}$ energetic shift (assuming equal oscillator strengths), which yields the transient shape and $f(t)$. For this measurement we derive an energetic shift of $+1.2 \mathrm{eV}$ and $f(300 \mathrm{ps})=9 \%$.

(ii) Relying on an energetic shift due to the change in oxidation state, we initially fitted the reactant state absorption spectrum in Fig. 2(a) to its main features: The intense $2 p_{3 / 2} \rightarrow 4 d\left(e_{g}\right)$ absorption (feature $B$ ) was reproduced using a Voigt profile with the Lorentz width taken from Ref. [19] including our spectral resolution of ca. $2.5 \mathrm{eV}$ (Gaussian width), an arctangent step function describing the edge absorption (together with higher lying states), and a second Voigt profile describing the $2 p_{3 / 2} \rightarrow 5 s_{1 / 2}$ transition (feature $C$ ). The fitted XAS spectrum shown in Fig. 2(c) (dashed) is very identical to the measured spectrum in Fig. 2(a). Next, we fitted the high-energy part of the transient in Fig. 2(b) to the difference spectrum of both energy-shifted $B \rightarrow B^{\prime}, C \rightarrow$ $C^{\prime}$ features together with the absorption edge. The fit result yields an energetic shift of about $1.2 \mathrm{eV}$ together with $f(300 \mathrm{ps})=11 \%$, which is very close to the above estimates. The resulting curve [dashed in Fig. 2(b)] matches the observed transient perfectly, except for the lower energy region. This part was then finally fitted assuming the appearance of a new absorption profile with Gaussian and Lorentzian widths of $2.5 \mathrm{eV}$ (monochromator resolution) and $1.9 \mathrm{eV} \mathrm{[19],} \mathrm{respectively,} \mathrm{which}$ arises from the photoinduced appearance of feature $A^{\prime}$. The solid curve in Fig. 2(b) now describes the measured transient XAS. The product state XANES $P(E, t)$, constructed via Eq. (1) with this transient fit curve, is shown in Fig. 2(c) (solid curve).

To date, no XAS exists for the intermediate species $\left[\mathrm{Ru}^{\mathrm{III}}\left(\mathrm{bpy}^{-}\right)(\mathrm{bpy})_{2}\right]^{2+}$ [solid curve in Fig. 2(c)], but we can compare our spectrum with the static XAS of the isoelectronic species $\left[\mathrm{Ru}^{\mathrm{III}}\left(\mathrm{NH}_{3}\right)_{6}\right] \mathrm{Cl}_{3}$ shown in Fig. 2(a). Indeed, the spectra are very similar in $A^{\prime}-B^{\prime}$ splitting $(3.8 \mathrm{eV})$ and $A^{\prime} / B^{\prime}$ relative intensities (ca. 2.5), which confirms the $3+$ oxidation state of the central Ru atom.

The loss of absorption near the maximum of feature $B$ [Fig. 2(b)] is shown in Fig. 4 as a function of time delay between the optical pump pulse and the $\mathrm{x}$-ray probe pulse. The temporal evolution can be described by a step function fitted with a Gaussian error function (solid curve). The signal buildup around time zero occurs within $96 \pm$ $30 \mathrm{ps}$, which indicates the cross correlation time between the ultrashort laser pulse and the $100 \mathrm{ps} \mathrm{x}$-ray pulse as shown by the (inverted) derivative of the fit curve in Fig. 4. Figure 4 represents a single $10 \mathrm{~min}$ scan, corresponding to 2500 pump-probe shots, thus utilizing 5000 $\mathrm{x}$-ray pulses at $2 \mathrm{kHz}$ per data point with our difference detection scheme.

The scans in Figs. 2 and 4 were recorded, respectively, with only $10^{7}$ and $10^{6}$ incident $\mathrm{x}$-ray photons per data 


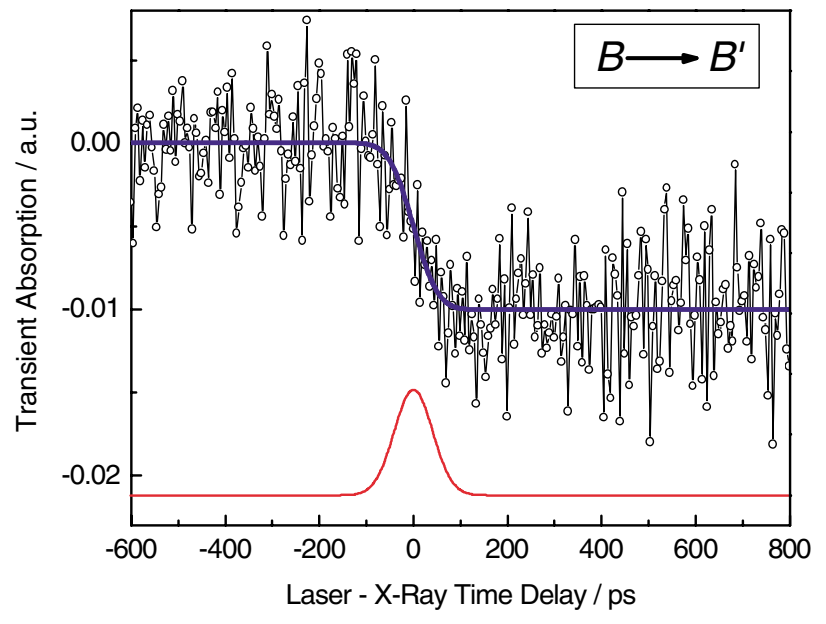

FIG. 4 (color online). Temporal evolution of the transient absorption measured near feature $B$ in Fig. 2(c) (at $2840.5 \mathrm{eV}$ ) together with a fit (solid curve). The inverted derivative of the fit curve (below) indicates the cross correlation time of ca. 100 ps governed by the $\mathrm{x}$-ray pulse width.

point, for ca. $10 \%$ of excited state species. However, the neat water solvent without solute already absorbs $90 \%$ of the incident flux $\left(\mathrm{H}_{2} \mathrm{O}\right.$ transmission at $2.8 \mathrm{keV}$ for $0.1 \mathrm{~mm}$ thickness). Thus the signal-to-noise of the transient signal would be identical to the one shown in Fig. 4 with only $10^{5}$ incident photons if we could neglect the solvent background absorption. This is promising for the future since most transition metal complexes have characteristic absorption edges above $5 \mathrm{keV}$, where solvent background losses are below $20 \%$, while the solute absorption can be optimized by increasing its concentration.

In summary, we have monitored a photoinduced charge transfer reaction of aqueous $\left[\mathrm{Ru}^{\mathrm{II}}(\mathrm{bpy})_{3}\right]^{2+}$ with $100 \mathrm{ps}$ temporal resolution using time-resolved XAS. While our findings are in full agreement with the expectations from optical spectroscopy experiments [2], to date this measurement describes the first detection of a change of oxidation state on the subnanosecond time scale by $\mathrm{x}$-ray absorption spectroscopy of a transient chemical species in solution. Hereby we have successfully resolved the excited state species in both x-ray absorption energy and in time. With data accumulation times similar to those of laser-only experiments, these results also show that time-resolved structural studies with EXAFS is within reach with present day picosecond $\mathrm{x}$-ray sources.

This work is funded by the Swiss National Science Foundation via Contracts No. 2000-059146.99 and No. 620-66145.01, by the Swiss Light Source (SLS), and by the Advanced Light Source (ALS). We thank O. Haas from PSI and R.W. Falcone from UC Berkeley for fruitful discussions, Dr. R.W. Schoenlein, Dr. M. Hertlein, Dr. A. Belkacem, and Dr. A. Lindenberg from the ALS for their assistance during the measurements.

*Corresponding author.

Email address: Christian.Bressler@ipmc.unil.ch

Corresponding author.

Email address: Majed.Chergui@ipmc.unil.ch

[1] T. S. Rose, M. J. Rosker, and A. H. Zewail, J. Chem. Phys. 91, 7415 (1989).

[2] A. Vlček, Chem. Rev. 200-202, 933 (2000).

[3] S. Franzen, L. Kiger, C. Poyart, and J. L. Martin, Biophys. J. 80, 2372 (2001).

[4] H. Z. Yu et al., Chem. Phys. Lett. 293, 1 (1998).

[5] H. B. Gray and J. Winkler, in Electron Transfer in Metalloproteins in Electron Transfer in Chemistry, edited by V. Balzani (Wiley-CH, Weinheim, 2001), Vol. III, p. 3.

[6] E. Lenderink et al., J. Phys. Chem. 100, 7822 (1996).

[7] F. M. F. de Groot et al., J. Chem. Phys. 101, 6570 (1994).

[8] C. Bressler et al., Proc. SPIE Int. Soc. Opt. Eng. 3451, 108 (1998).

[9] F. L. H. Brown, K. R. Wilson, and J. Cao, J. Chem. Phys. 111, 6238 (1999).

[10] I. V. Tomov, D. A. Oulianov, P. Chen, and P. M. Rentzepis, J. Phys. Chem. B 103, 7081 (1999).

[11] C. Bressler et al., J. Chem. Phys. 116, 2955 (2002).

[12] C. Bressler et al., in Femtochemistry and Femtobiology, edited by A. Douhal and J. Santamaria (World Scientific, Singapore, 2002), p. 449.

[13] D. J. Thiel, P. Livins, E. A. Stern, and A. Lewis, Nature (London) 362, 40 (1993).

[14] D. M. Mills, A. Lewis, A. Harootunian, J. Huang, and B. Smith, Science 223, 811 (1984).

[15] H. X. Wang, et al., J. Am. Chem. Soc. 119, 4921 (1997).

[16] L. X. Chen et al., Science 292, 262 (2001).

[17] F. Ráksi et al., J. Chem. Phys. 104, 6066 (1996).

[18] N. H. Damrauer et al., Science 275, 54 (1997).

[19] T. K. Sham, J. Chem. Phys. 83, 3222 (1985).

[20] C. Sigiura, M. Kitamura, and S. Muramatsu, J. Chem. Phys. 84, 4824 (1986). 